\title{
Maternity service organisational interventions that aim to reduce caesarean section: a systematic review and meta- analyses
}

Anna Chapman ${ }^{1,2}$, Cate Nagle ${ }^{3,4}$, Debra Bick ${ }^{5}$, Rebecca Lindberg ${ }^{6}$, Bridie Kent ${ }^{7}$, Justin Calache ${ }^{1,2}$ and Alison M. Hutchinson ${ }^{1,2^{*}}$ (D)

\begin{abstract}
Background: Caesarean sections (CSs) are associated with increased maternal and perinatal morbidity, yet rates continue to increase within most countries. Effective interventions are required to reduce the number of non-medically indicated CSs and improve outcomes for women and infants. This paper reports findings of a systematic review of literature related to maternity service organisational interventions that have a primary intention of improving CS rates.

Method: A three-phase search strategy was implemented to identify studies utilising organisational interventions to improve CS rates in maternity services. The database search (including Cochrane CENTRAL, CINAHL, MEDLINE, Maternity and Infant Care, EMBASE and SCOPUS) was restricted to peer-reviewed journal articles published from 1 January 1980 to 31 December 2017. Reference lists of relevant reviews and included studies were also searched. Primary outcomes were overall, planned, and unplanned CS rates. Secondary outcomes included a suite of birth outcomes. A series of metaanalyses were performed in RevMan, separated by type of organisational intervention and outcome of interest. Summary risk ratios with 95\% confidence intervals were presented as the effect measure. Effect sizes were pooled using a randomeffects model.

Results: Fifteen articles were included in the systematic review, nine of which were included in at least one meta-analysis. Results indicated that, compared with women allocated to usual care, women allocated to midwife-led models of care implemented across pregnancy, labour and birth, and the postnatal period were, on average, less likely to experience CS (overall) (average RR 0.83, 95\% Cl 0.73 to 0.96), planned CS (average RR 0.75, 95\% Cl 0.61 to 0.93), and episiotomy (average RR $0.84,95 \%$ Cl 0.74 to 0.95 ). Narratively, audit and feedback, and a hospital policy of mandatory second opinion for CS, were identified as interventions that have potential to reduce CS rates.

Conclusion: Maternity service leaders should consider the adoption of midwife-led models of care across the maternity episode within their organisations, particularly for women classified as low-risk. Additional studies are required that utilise either audit and feedback, or a hospital policy of mandatory second opinion for CS, to facilitate the quantification of intervention effects within future reviews.
\end{abstract}

PROSPERO registration: CRD42016039458; prospectively registered.

Keywords: Caesarean section, Systematic review, Meta-analysis, Organisational interventions, Childbirth, Maternity service, Midwife-led care

\footnotetext{
* Correspondence: alison.hutchinson@deakin.edu.au

'School of Nursing and Midwifery, Centre for Quality and Patient Safety

Research, Deakin University, Geelong, VIC, Australia

${ }^{2}$ Monash Medical Centre, Monash Health, Level 2 I Block, 246 Clayton Rd,

Clayton 3168, VIC, Australia

Full list of author information is available at the end of the article
}

(c) The Author(s). 2019 Open Access This article is distributed under the terms of the Creative Commons Attribution 4.0 International License (http://creativecommons.org/licenses/by/4.0/), which permits unrestricted use, distribution, and reproduction in any medium, provided you give appropriate credit to the original author(s) and the source, provide a link to the Creative Commons license, and indicate if changes were made. The Creative Commons Public Domain Dedication waiver (http://creativecommons.org/publicdomain/zero/1.0/) applies to the data made available in this article, unless otherwise stated. 


\section{Background}

Caesarean section (CS), when medically indicated, can effectively prevent maternal and perinatal morbidity and mortality [1]. However, steady increases in CS rates within the majority of regions worldwide have generated concern about the utilisation of this procedure when not medically justified [2]. The average CS growth rate among Organisation for Economic Cooperation and Development (OECD) countries increased from $20 \%$ in 2000 to $28.6 \%$ in 2016, with the highest rates observed in Turkey, Chile and Mexico ( $\geq 46 \%)$ [3]. Recent epidemiological research has indicated that population-level CS rates above 19\% are not associated with reductions in maternal and neonatal mortality $[1,4]$. Consequently, appropriate utilisation of CS is a central focus for health professionals and health systems globally, with equitable access and patient safety considered paramount.

Global concern for patient safety in response to rising rates of CS is warranted. While CS effectively expedites birth in obstetric emergencies, CSs when not medically indicated, are associated with an increased risk of short- and long-term complications for women and their infants. For women, a CS can increase the risk of bladder injuries, postpartum infections, anaesthetic complications, obstetric shock, hysterectomy, thromboembolism and psychological distress [5]. In contrast, benefits of planned vaginal birth, compared with planned CS birth among low-risk women, include lower rates of infection and faster recovery [6]. For the term infant, medically unjustified CSs have been associated with increased risk of neonatal intensive care admission and respiratory problems, and a reduced likelihood of breastfeeding initiation [7, 8]. The economic burden of CSs is also worth noting. Compared with vaginal delivery, higher mean costs have been associated with CS in low-risk populations [9].

A multitude of reasons are linked to the increasing trend in CS rates particularly within high-resource settings, including a shift in maternal socio-demographic characteristics, scheduling convenience, changes to professional practice styles, an increase in pre-existing maternal medical conditions (i.e. diabetes, obesity), malpractice liability concerns, and an enhanced maternal preference for the procedure [10]. These factors have been targeted in a variety of interventions designed to safely reduce CS rates in high-use settings. To date, the effectiveness of interventions directed at both women and healthcare providers is limited, with very few studies displaying clinically meaningful effects [11]. Organisational interventions, on the other hand, have recently been posited as having the most potential for reducing the rising trend in CS rates [12]. As outlined by Cochrane Effective Practice and Organisation of Care Group, an organisational intervention is one which "...involves a change in the structure or delivery of health care
... a change in who delivers health care, how care is organised, or where care is delivered..." [13]. Not since 2007 has a systematic review of maternity service organisational interventions been performed; in this review, positive effects on CS rates were observed for audit and feedback, quality improvement, and multifaceted strategies (e.g. combination of education, audit and feedback and implementation of practice guidelines) [14]. Additionally, two recent Cochrane reviews have examined the effects of midwife-led models of care [15] and oneto-one support in labour [16] on CS, with positive effects observed for one-to-one labour support only. These reviews did not specifically limit inclusion to interventions with a primary outcome of CS; furthermore, the review of one-to-one support in labour included studies that utilised informal support persons within interventions. It is therefore timely that a systematic review of organisational interventions specifically designed to improve CS rates in maternity services be performed.

This systematic review and meta-analyses was designed to synthesise literature related to maternity service organisational interventions that had a primary aim of improving CS rates. Additionally, this work sought to quantify the effectiveness of relevant organisational interventions on CS rates, relative to comparator conditions. Findings of this review have potential to inform future policies and programs designed to optimise the utilisation of CS within maternity service settings.

\section{Methods}

This systematic review complies with the Preferred Reporting Items for Systematic reviews and Meta-Analyses (PRISMA) statement [17]. The protocol for the review was prospectively registered in PROSPERO (CRD420160 39458), and the study protocol, describing the rationale and methods in detail, has been published [18].

\section{Data sources and search strategy}

A three-phase search strategy was implemented to identify literature relevant to the current investigation. Firstly, electronic databases were searched, structured according to the nuances for each database (the Cochrane Central Register of Controlled Trials, CINAHL, MEDLINE, Maternity and Infant Care, EMBASE and SCOPUS) using a combination of keywords relating to CS and maternity service organisational interventions and controlled vocabulary. The search was restricted to peer-reviewed, journal articles published from 1 January 1980 to 31 December 2017. No restrictions on language or setting were applied. An example of the MEDLINE search strategy is included as a Additional file 1. Secondly, reference lists of relevant reviews captured within the database search were manually cross-checked to identify additional articles. Thirdly, reference lists of all included studies were reviewed to identify additional eligible 
references. Two reviewers independently assessed titles and abstracts of studies for inclusion at all three stages. Selections were then compared and where discrepancies occurred, consensus was reached through discussion with a third reviewer.

\section{Eligibility criteria}

Studies were only included if they reported on maternity service organisational interventions designed to reduce CS rates (including planned and unplanned CS). Eligible study designs were those classified as randomised controlled trials (RCTs), cluster-RCTs, quasi-RCTs, controlled before and after studies, and interrupted time series studies. Inclusion criteria were based on the following PICO (participants, interventions, comparators, outcomes) criteria:

- Participants and settings: Maternity care clinicians, including midwives, obstetricians, nurses, paediatricians, family doctors and anaesthetists, maternity care managers and maternity care educators. Eligible settings were obstetric-led maternity services able to provide support for women undergoing planned or unplanned caesarean birth.

- Interventions: Eligible maternity service organisational interventions included models of care, audit and feedback, hospital policy/protocol interventions, labour assessment triage, incentives, education, and reminder mechanisms. Intervention strategies aiming to reduce CS rates were permitted to be either single component, or multi-faceted (i.e. complex interventions).

- Comparators: An appropriate comparator group was specified as no intervention, or usual/routine care.

- Outcomes: Studies that reported objectively measured or self-reported (using validated instruments) outcomes. Planned, unplanned and overall CS rates were the primary outcome measures. Studies that did not specify CS rates as the primary outcome measure were not eligible for inclusion. Where studies did not explicitly differentiate between primary and secondary outcome measures, studies were included if CS rate was deemed by the systematic review authors as an outcome of priority. Secondary outcomes of interest included labour interventions (e.g. epidural use, labour augmentation), maternal adverse events (e.g. postpartum haemorrhage, third or fourth degree perineal tear), neonatal outcomes (e.g. admission to neonatal intensive care unit, Apgar scores), breastfeeding initiation, maternal/newborn duration of inpatient stay, maternal experiences of care, adherence to best practice guidelines by health professionals, health professionals' satisfaction, confidence, competence, attitudes, knowledge and self-efficacy.
Medical interventions (e.g. induction of labour, episiotomy, instrumental vaginal delivery), lifestyle interventions (e.g. nutrition and physical activity programs for pregnant women), labour interventions (e.g. water births, epidural analgesia, augmentation of labour), and interventions utilising active management of labour, were not deemed eligible maternity service organisational interventions and were excluded. Additionally, doulas (also referred to as birth companions) and informal support persons were not classified as eligible health care providers in this review; hence, any studies targeting these providers were also excluded.

\section{Data extraction}

Two reviewers independently extracted data from included studies using a standardised template. The resultant data included study and sample characteristics (e.g. research aim, setting, sample size), design features (e.g. intervention type/s and regimen of intervention condition/s) and study results (e.g. RR, 95\% CI). Only data reported in the original papers were used for extraction, and no attempts were made to contact corresponding authors to obtain unpublished data.

\section{Risk of bias/quality appraisal}

The Cochrane Collaboration's tool for assessing risk of bias was used for all included studies that utilised an RCT or cluster RCT design [19]. This tool examines randomisation procedure and allocation concealment (selection bias); blinding of participants and personnel (performance bias); blinding of outcome assessors (detection bias); incomplete outcome data (attrition bias); selective outcome reporting (reporting bias); and other sources of bias (e.g. baseline imbalance, recruitment issues etc.). A positive classification indicated a low risk of bias, while a negative classification indicated a high risk of bias. An unclear classification was given when there was insufficient information within manuscripts to adequately assess risk of bias.

The Quality Assessment Tool for Quantitative Studies [20] was used for all other research designs. This tool addresses eight domains: selection bias; study design; confounders; blinding; data collection; participant withdrawals; intervention integrity and analysis. The quality assessment across the eight domains allows an overall quality rating to be determined for each study: 'strong,' 'moderate' or 'weak'.

Two reviewers independently performed all risk of bias/ quality appraisal assessments, with consensus reached through joint discussion.

\section{Data synthesis and analysis}

For studies utilising an RCT design, a series of metaanalyses were performed that were separated by type of maternity service organisational intervention and outcome of interest. A minimum of three studies per outcome was considered adequate for a meta-analysis. Where a meta- 
analysis was not possible (e.g. $<3$ studies per outcome per intervention type), the results were synthesised and discussed narratively.

Quantitative data from included studies were analysed using RevMan 5.3 software [21]. Secondary outcome variables reported within included studies were mapped to assist with the selection and prioritisation of secondary outcomes to be reported in this review. Secondary outcomes were included in this review when a minimum of three included studies reported on a given outcome. All meta-analyses performed involved the use of dichotomous data, and as such, summary risk ratio with $95 \%$ confidence intervals were presented as the effect measure. Effects were pooled using a random-effects model (Mantel-Haenszel method). In contrast to a fixed-effects model that assumes all included studies share one true effect, a random-effects model assumes each study estimates a different underlying true effect, and produces a summary effect that is an estimate of the mean of a distribution of true effects [22]. After obtaining the full set of included studies, and noting the substantial clinical and methodological heterogeneity between studies (i.e. differences in intervention regimens, country of origin), a random-effects model was selected in preference to a fixed-effects model, as it was deemed unreasonable to assume that all included studies shared a common true effect.

Heterogeneity in each meta-analysis was examined using the $\mathrm{I}^{2}, \mathrm{Tau}^{2}$, and $\mathrm{X}^{2}$ statistic with associated significance value. A $p$-value for the $\chi^{2}$ statistic of 0.10 (rather than $0.05)$ was used to indicate statistically significant heterogeneity due to the limitations of this test when there are low numbers of included studies [23, 24]. The following classifications for the $\mathrm{I}^{2}$ statistic were used to determine the degree of heterogeneity: not important (0-40\%), moderate heterogeneity (30-60\%), substantial heterogeneity (5090\%) and considerable heterogeneity (75-100\%) [23]. Tau ${ }^{2}$ values were examined and subsequently reported within the text when heterogeneity was found to be significant, or the $\mathrm{I}^{2}$ statistic was $\geq 30 \%$.

Subgroup analyses to examine potential sources of heterogeneity were pre-specified that utilised the Robson classification system [25] to differentiate between interventions by type of maternal group [18]. This planned assessment was not possible however, as included studies utilised maternal participant groups that were either not described in sufficient detail or encompassed a diverse mix of maternal groups (not separated in analysis). Similarly, an assessment of publication bias/small study effects could not be performed due to the limited number of included studies ( $<10$ studies).

It was initially planned to combine results from cluster RCTs with individually-randomised RCTs if there was minimal clinical and methodological heterogeneity between the studies, and the interaction between the intervention effect and choice of randomisation units were deemed unlikely [18]. However, only RCTs with a parallel design were ultimately eligible for inclusion within the quantitative synthesis. Had any cluster RCTs been eligible for a least one metaanalysis, the standard error of the cluster RCT would have been adjusted using the reported intracluster correlation coefficient. Heterogeneity in the unit of randomisation would also have been acknowledged, and a sensitivity analysis to investigate the effects of the randomisation unit would have been performed.

\section{Results}

The three-phase search strategy yielded 11,586 citations after duplicates were removed. The first phase of screening identified 171 abstracts as potentially eligible; full texts were subsequently retrieved and assessed for inclusion. One hundred and fifty-six articles were excluded in the second phase of screening as they did not meet specified criteria. In total, 15 articles were included in the systematic review, nine of which were appropriate for inclusion in at least one meta-analysis. A PRISMA flow diagram details the results of the systematic search and screening process (Fig. 1).

The characteristics of included studies are provided in Table 1 . The majority of studies utilised a parallel RCT design $(\mathrm{k}=12,80 \%)$, with the remaining studies adopting either a cluster RCT design $(k=2,13 \%)[26,27]$, or quasiexperimental controlled design $(k=1,7 \%)$ [28]. Year of publication ranged from 1992 to 2015, with the majority of studies published from $2001(\mathrm{k}=11,73 \%)$. All studies were published in English, and all interventions were implemented in hospitals; however, countries varied: USA/ Canada $(\mathrm{k}=6,40 \%)$, Australia $(\mathrm{k}=4,27 \%)$, Iran $(\mathrm{k}=2$, $13 \%)$, Ireland $(\mathrm{k}=1,6.7 \%)$, China $(\mathrm{k}=1,6.7 \%)$, and Latin America $(k=1,6.7 \%)$. Birth outcome data were largely obtained via medical record audits for samples of women, with samples ranging in size from 100 to 149,276 participants (mean 14,665 $\pm 39,509$; median 1172). Midwife-led models of care were the most commonly utilised maternity service organisational intervention $(\mathrm{k}=8,53 \%)$, which were primarily implemented across pregnancy, labour, birth, and the postnatal period (postnatal periods ranged from early through to 6-weeks), $(\mathrm{k}=6)$. In the six studies where the midwife-led model of care traversed all maternal periods, two studies utilised a caseload approach [29,30], with the other four studies using a team-based approach [31-34]. Other maternity service organisational interventions utilised included continuous midwifery care (also referred to as one-to-one/continuous labour support) $(\mathrm{k}=3,20 \%)$ [35-37], audit and feedback to promote implementation of evidence-based practice $(k=1,6.7 \%)$ [27], labour assessment triage (at home vs hospital) $(\mathrm{k}=1,6.7 \%)$ [38], hospital policy of mandatory second opinion for CS $(k=1$, 6.7\%) [26], and hospital protocols for management of pregnancy complications $(\mathrm{k}=1,6.7 \%)$ [28]. In the majority of 


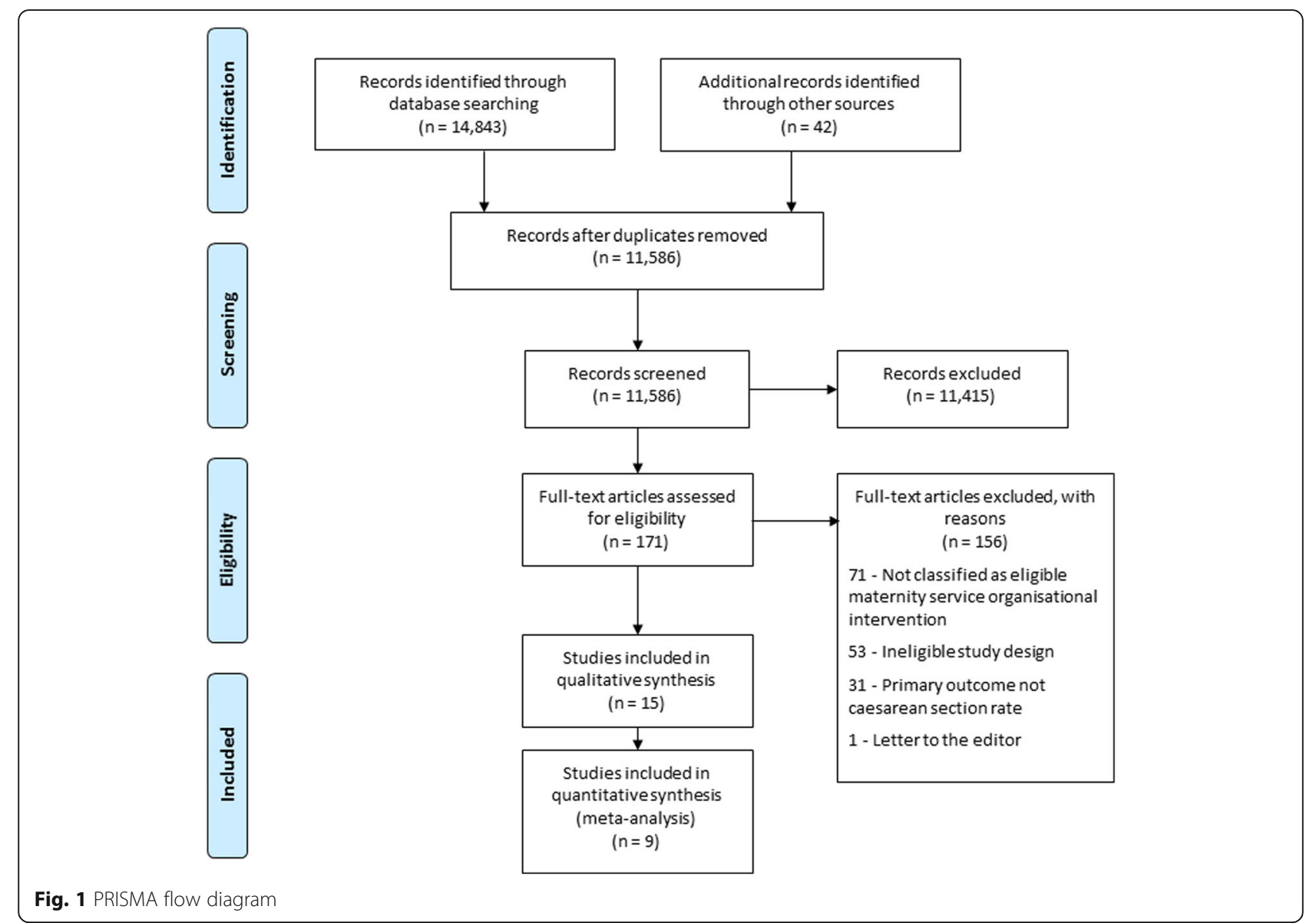

studies, these additional types of organisational intervention were implemented during labour and birth $(k=5)$, while in one study the intervention was implemented only during pregnancy [28].

Results of this systematic review are grouped and reported according to the type of maternity service organisational intervention. The presentation of results for primary outcomes have been prioritised, followed by secondary outcomes. The data for meta-analyses are presented first, followed by the narrative presentation of results. The estimated effects and heterogeneity of meta-analyses are summarised in Table 2. With regard to the narrative synthesis of outcomes, a small portion of secondary outcomes (maternal/newborn duration of inpatient stay, maternal experiences of care, adherence to best practice guidelines by health professionals, and health professionals' satisfaction, confidence, competence, attitudes, knowledge and selfefficacy) were not reported in enough studies $(<3$ of all included studies) to warrant inclusion.

\section{Midwife-led models of care}

In the present review, a midwife-led model of care was defined as care where "the midwife is the lead professional in the planning, organisation and delivery of care given to a woman" [15]. A midwife-led model of care was implemented within eight included studies [29-34, 39, 40]. Six of these studies [29-34] were similar in terms of intervention regimen (midwife-led vs usual care), maternal period of study (pregnancy, labour and birth, and postnatal), and study design (RCT), and as such, results of these studies were pooled in a series of separate meta-analyses.

\section{Overall caesarean sections}

Six studies ( $n=7784$ participants) were included in a meta-analysis comparing midwife-led models of care (delivered over pregnancy, labour and birth, and postnatal periods) with a comparator group for overall CSs. Women allocated to midwife-led care were, on average, less likely to have a CS (average RR $0.83,95 \%$ CI 0.73 to 0.96) (Fig. 2). The $x 2$ test for heterogeneity was not significant, with heterogeneity considered not important to moderate $\left(\mathrm{X}^{2}=8.32, p=0.14, \mathrm{I}^{2}=40 \%, \mathrm{Tau}^{2}=0.01\right)$. Narratively, midwife-led models of care were utilised within two additional studies that assessed overall CS rates. One RCT, implemented in the USA, compared the effects of midwife-led labour and birth care provided in a birth centre with routine labour and birth care provided 


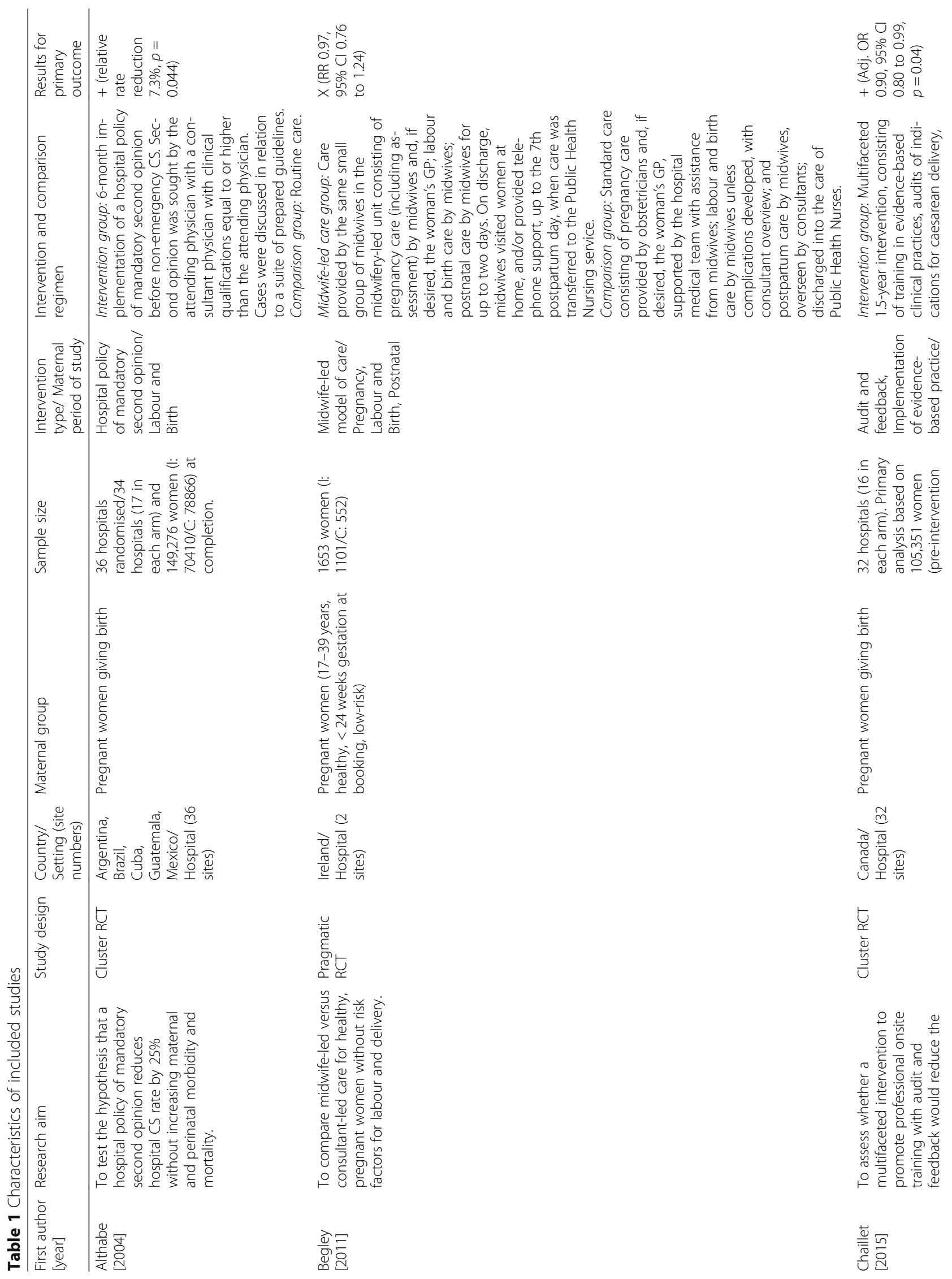




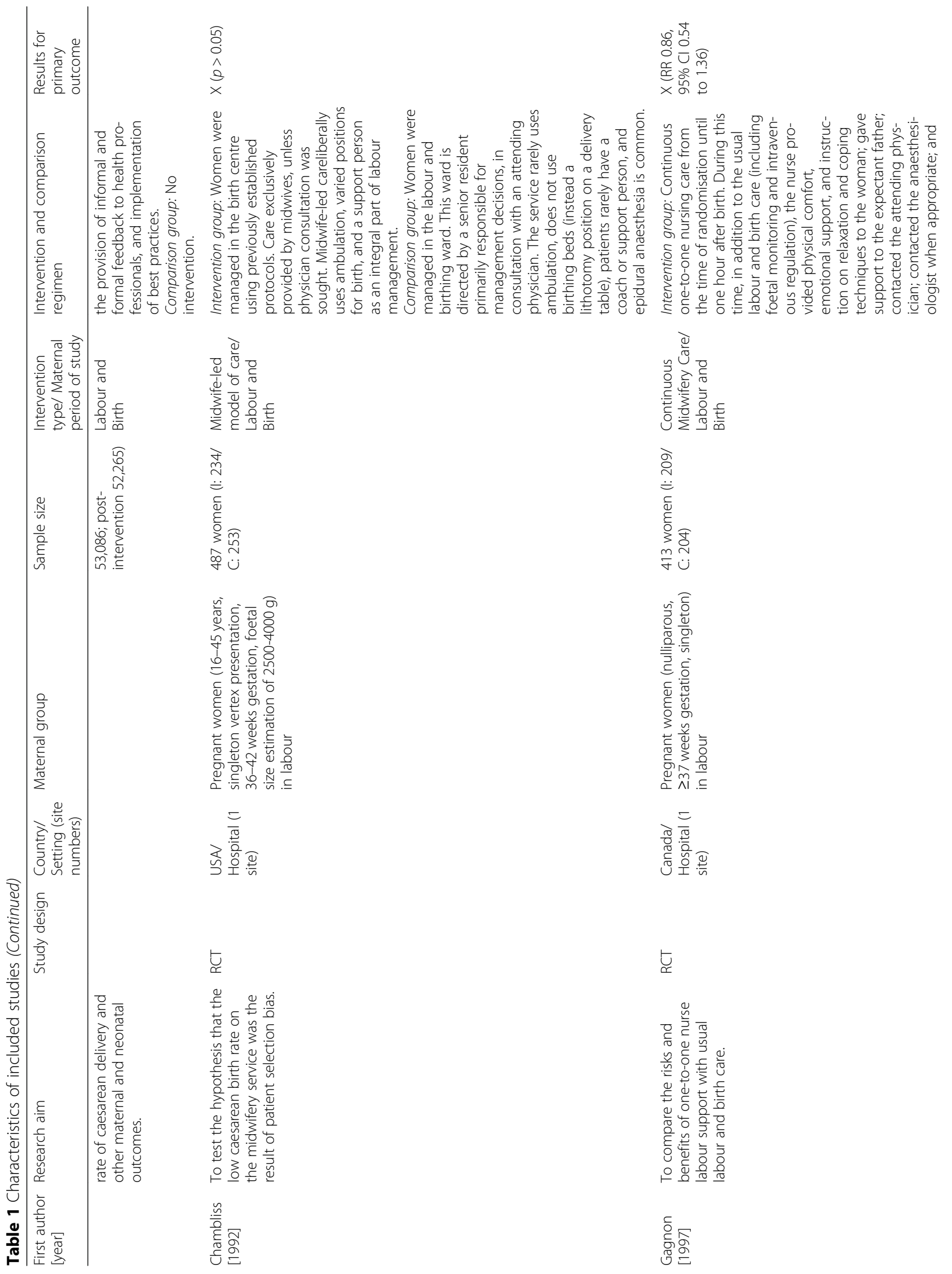




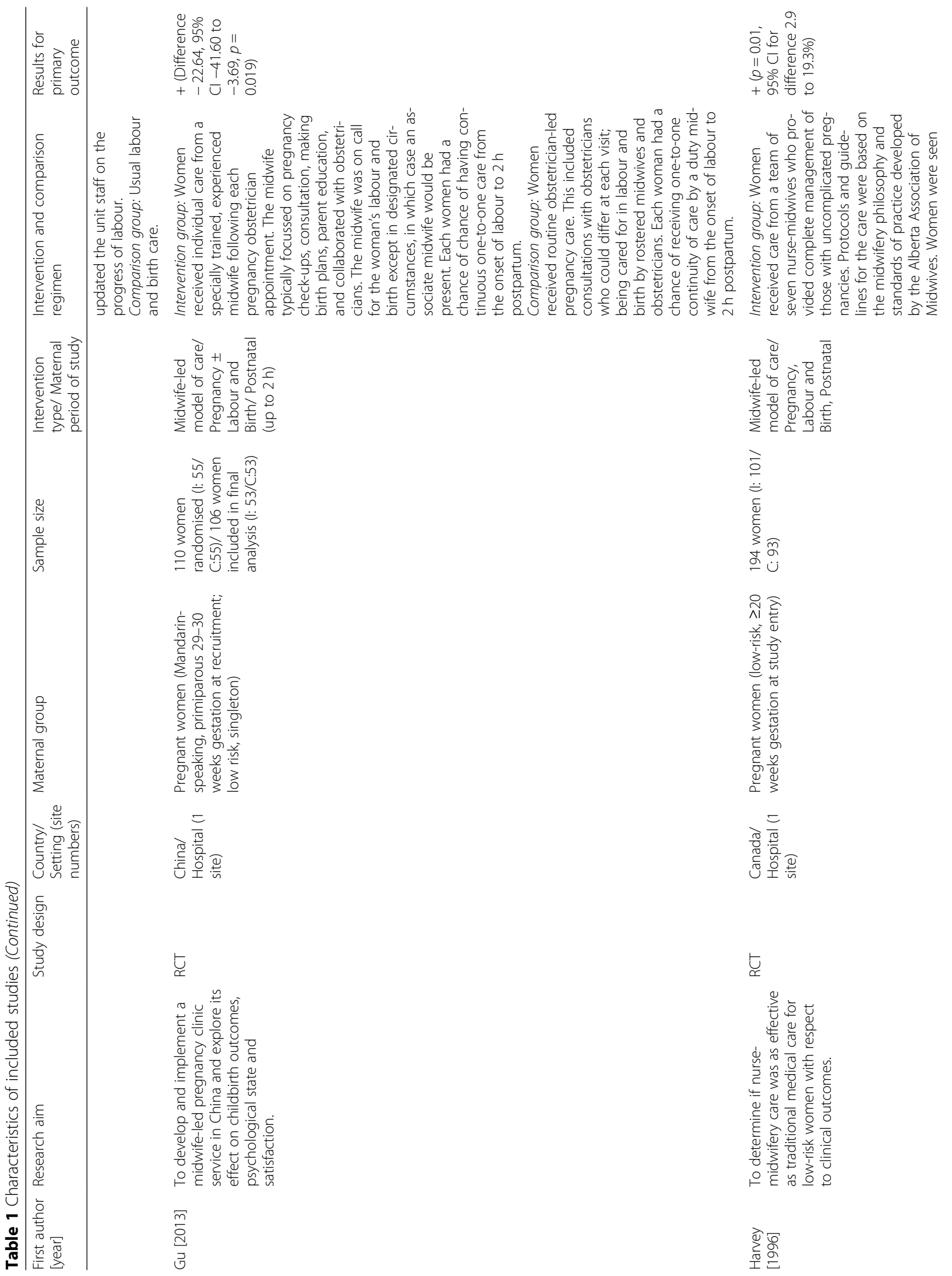




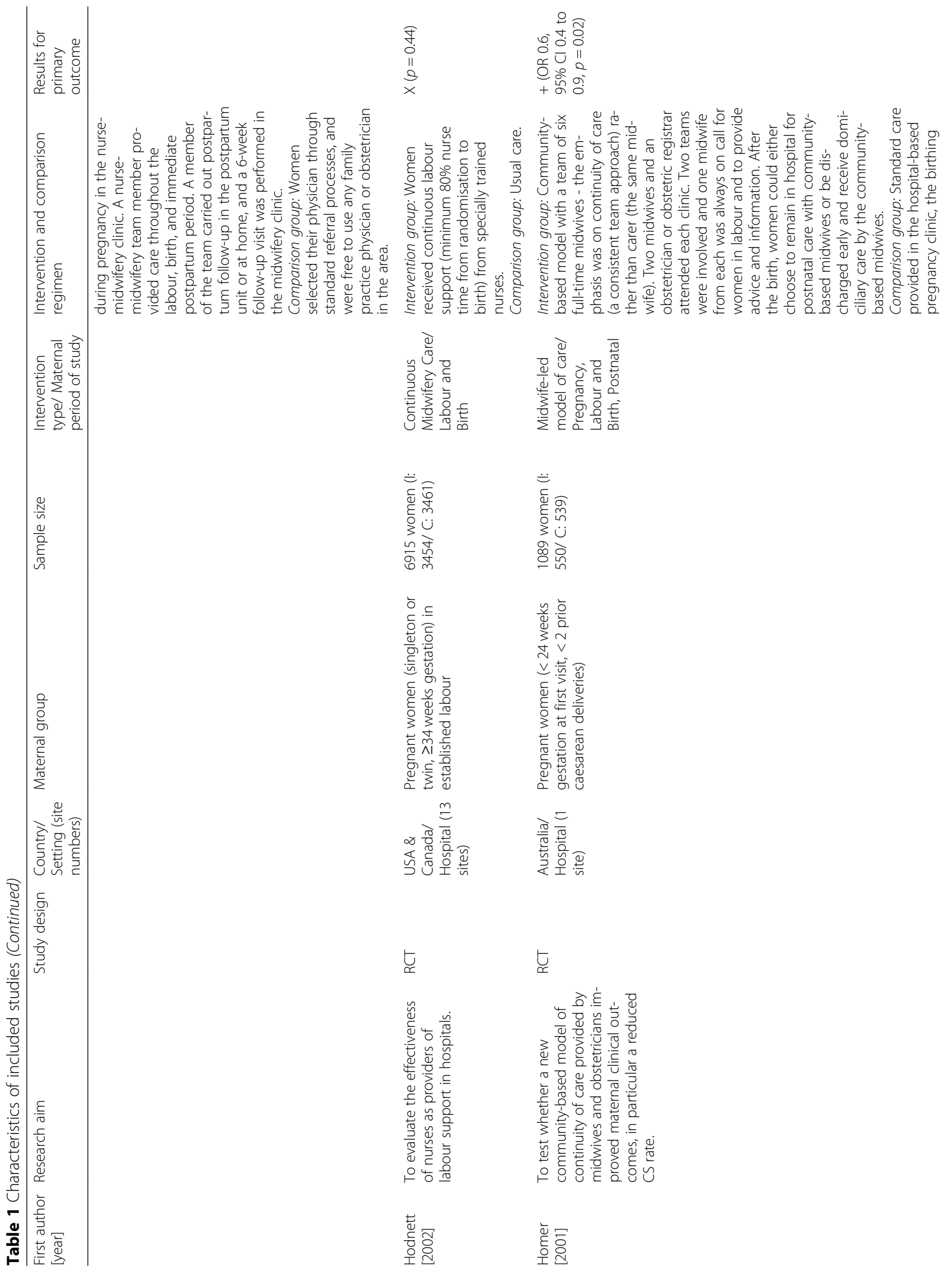




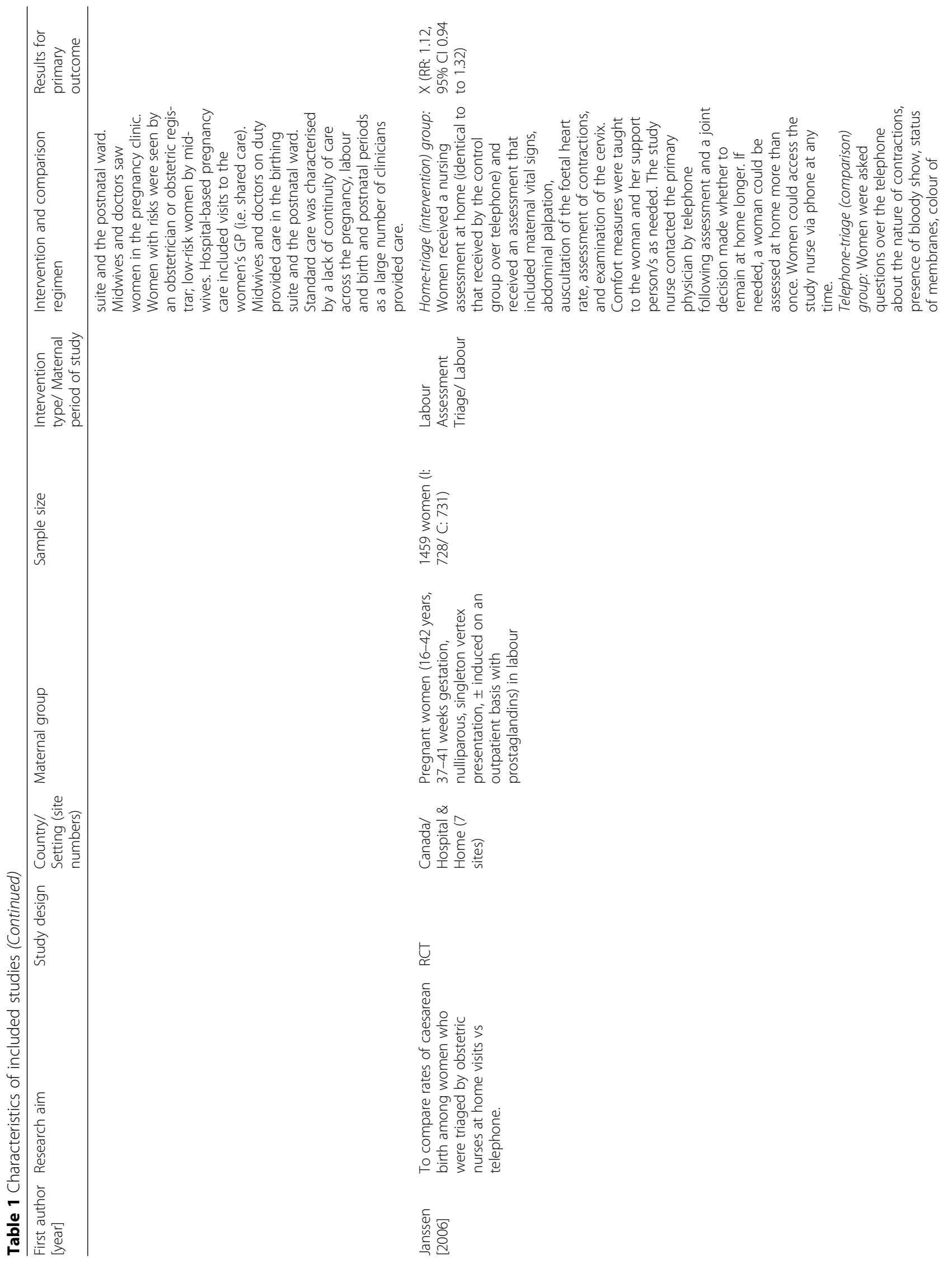




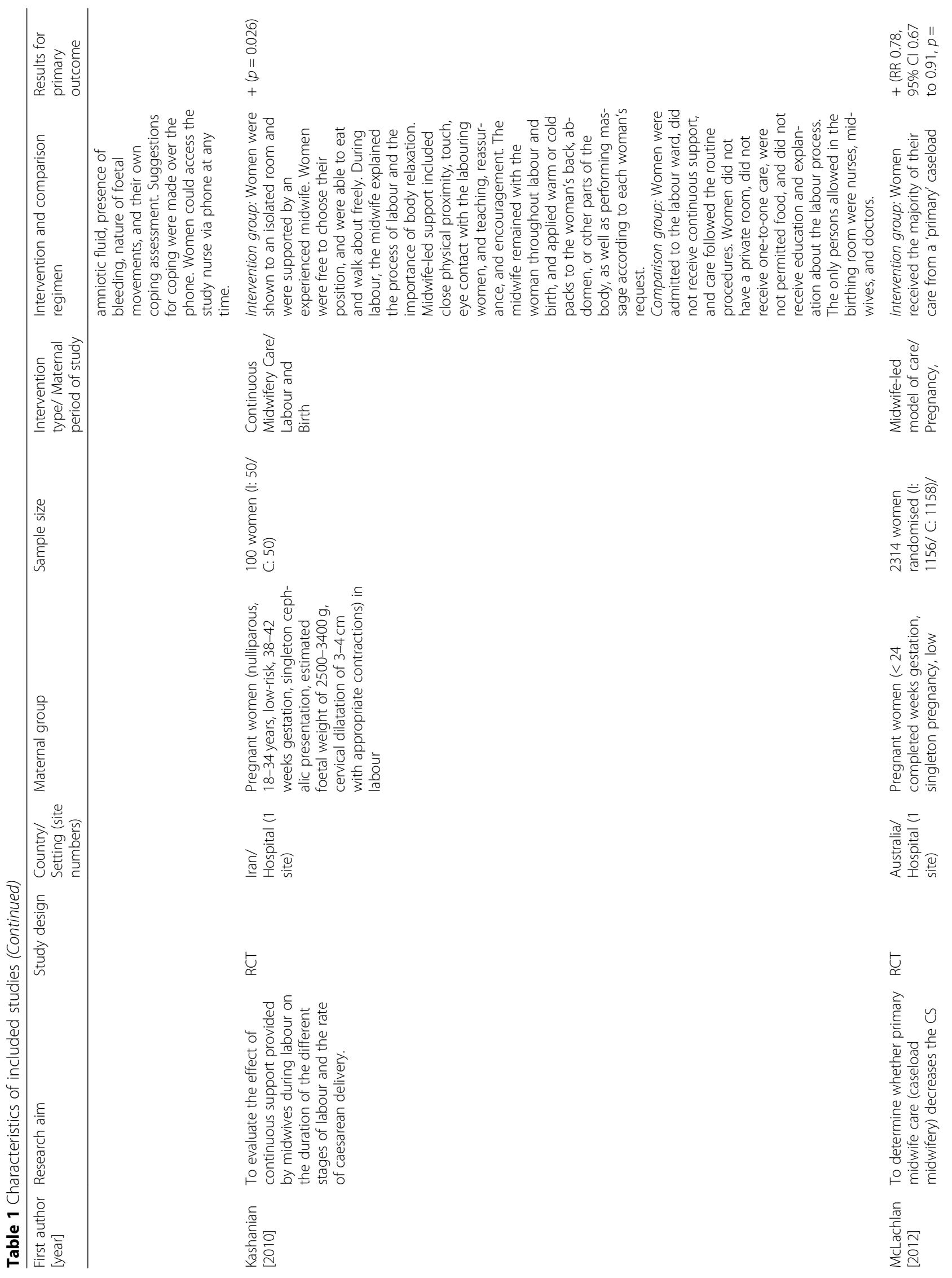




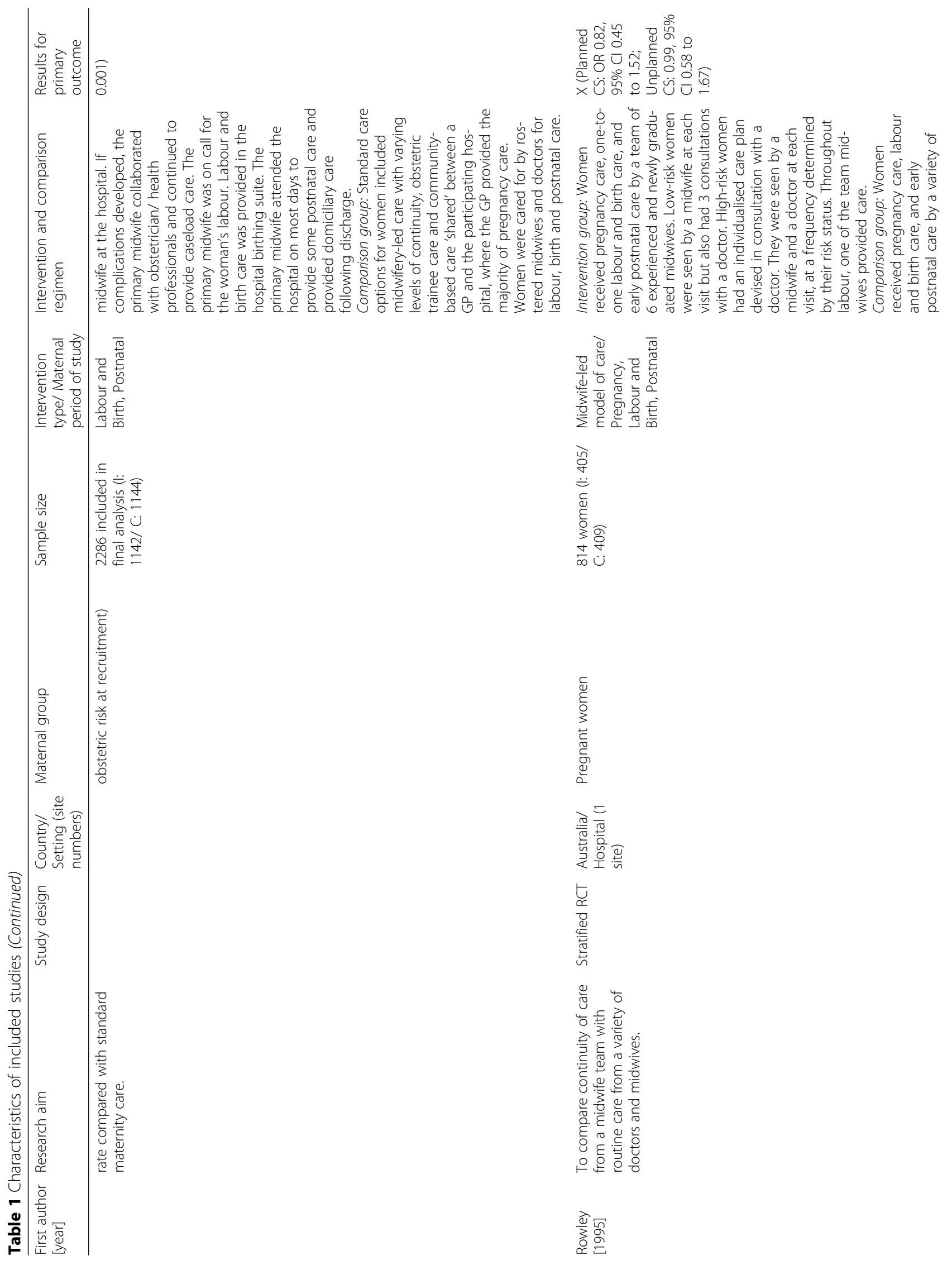




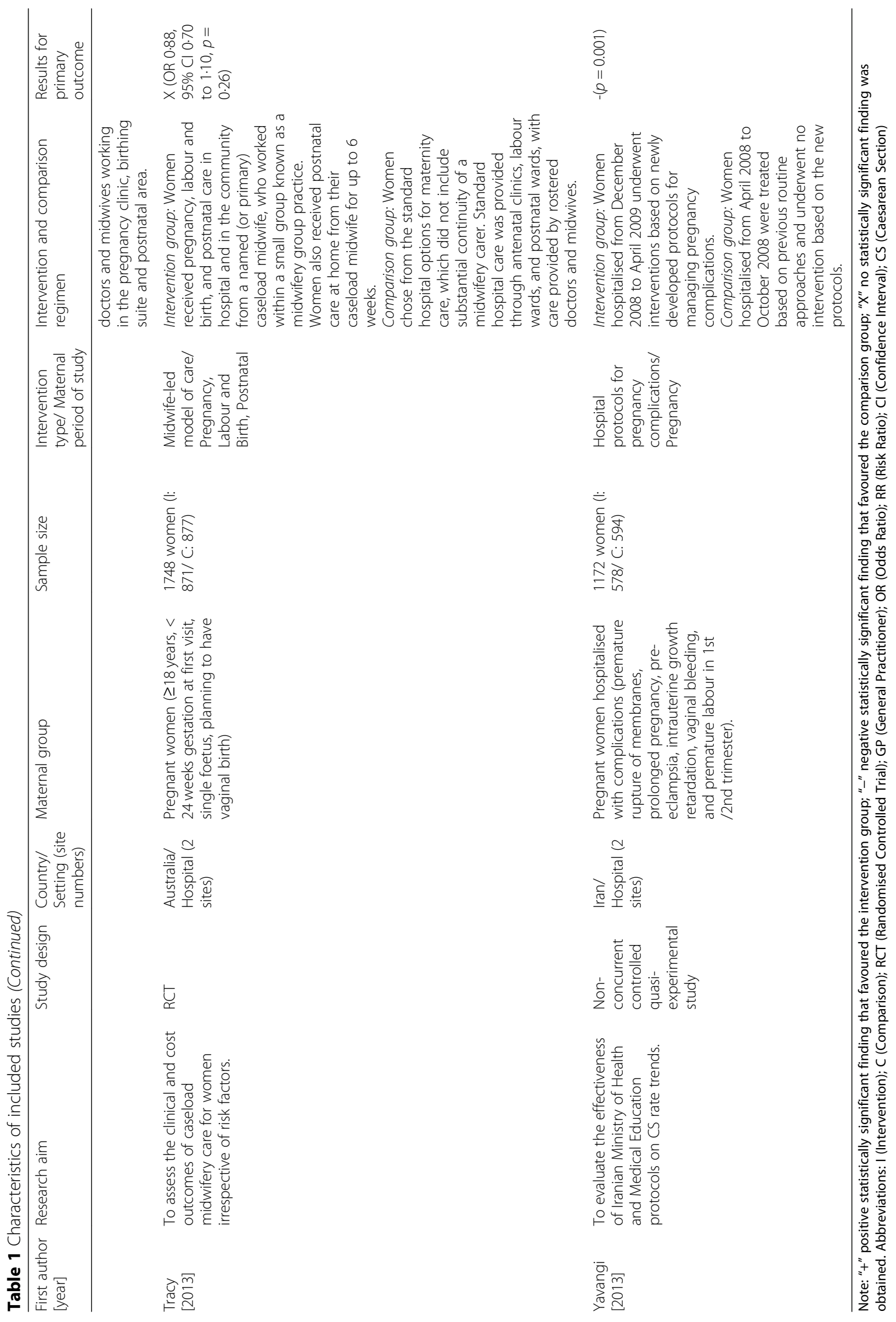


Table 2 Summary of meta-analyses

\begin{tabular}{|c|c|c|c|c|c|c|c|c|c|}
\hline \multirow[t]{2}{*}{ Outcomes } & \multirow[t]{2}{*}{ K } & \multirow[t]{2}{*}{$\mathrm{N}$} & \multicolumn{3}{|c|}{ Effect estimate } & \multicolumn{4}{|c|}{ Heterogeneity } \\
\hline & & & $\overline{\mathrm{RR}}$ & $95 \% \mathrm{Cl}$ & $Z(p)$ & $\overline{x^{2}}$ & $p$ & $P^{2}$ & $\mathrm{Tau}^{2}$ \\
\hline \multicolumn{10}{|l|}{ Midwife-Led Care vs. Comparator } \\
\hline Overall Caesarean Section & 6 & 7784 & 0.83 & 0.73 to 0.96 & $2.63(0.008)$ & 8.32 & 0.14 & $40 \%$ & 0.01 \\
\hline Planned Caesarean Section & 4 & 5937 & 0.75 & 0.61 to 0.93 & $2.66(0.008)$ & 1.19 & 0.76 & $0 \%$ & 0.00 \\
\hline Unplanned Caesarean Section & 4 & 5937 & 0.87 & 0.73 to 1.03 & $1.65(0.10)$ & 4.55 & 0.21 & $34 \%$ & 0.01 \\
\hline Induction of Labour & 5 & 5498 & 0.91 & 0.79 to 1.04 & $1.43(0.15)$ & 6.06 & 0.19 & $34 \%$ & 0.01 \\
\hline Epidural & 6 & 7601 & 0.89 & 0.79 to 1.00 & $1.96(0.05)$ & 10.82 & 0.06 & $54 \%$ & 0.01 \\
\hline Labour Augmentation & 5 & 5498 & 0.97 & 0.73 to 1.29 & $0.23(0.81)$ & 48.99 & $<0.00001$ & $92 \%$ & 0.09 \\
\hline Instrumental Vaginal Delivery & 4 & 6776 & 0.96 & 0.86 to 1.07 & $0.78(0.44)$ & 1.82 & 0.61 & $0 \%$ & 0.00 \\
\hline Episiotomy & 6 & 6816 & 0.84 & 0.74 to 0.95 & $2.87(0.004)$ & 6.19 & 0.29 & $19 \%$ & 0.00 \\
\hline Admission during Pregnancy & 4 & 5304 & 0.94 & 0.80 to 1.11 & $0.77(0.44)$ & 7.06 & 0.07 & $58 \%$ & 0.02 \\
\hline Apgar scores (<7 at $5 \mathrm{~min})$ & 3 & 4711 & 0.94 & 0.66 to 1.33 & $0.37(0.71)$ & 0.88 & 0.64 & $0 \%$ & 0.00 \\
\hline Admission to SCU/NICU & 5 & 6599 & 0.80 & 0.62 to 1.04 & $1.66(0.10)$ & 8.54 & 0.07 & $53 \%$ & 0.04 \\
\hline \multicolumn{10}{|c|}{ Continuous Midwifery Care vs. Comparator } \\
\hline Overall Caesarean Section & 3 & 7428 & 0.85 & 0.59 to 1.23 & $0.88(0.38)$ & 4.27 & 0.12 & $53 \%$ & 0.06 \\
\hline
\end{tabular}

Abbreviations: $K$ number of studies, $N$ number of participants, $R R$ Risk Ratio, $C l$ Confidence Interval, $Z$ test for overall effect

in a labour ward. This study observed no significant difference in overall CSs between groups (midwife-led: 5/ 234; routine: $1 / 253 ; p>0.05$ ) [39]. The other study, a RCT conducted in China, compared the effects of a midwifeled pregnancy clinic with routine obstetrician-led pregnancy care. This study found that women allocated to the intervention group were less likely to have a CS compared with women in obstetrician-led care group (I: 18/53; C: 30/53; 95\% CI for difference -41.60 to -3.69 ) [40].

\section{Planned caesarean section}

Four studies ( $n=5937$ participants) were included in a meta-analysis comparing midwife-led models of care with a comparator group for planned CSs. Women allocated to midwife-led care were, on average, less likely to experience a planned CS (average RR 0.75, 95\% CI 0.61 to 0.93) (Fig. 3)
. The $\chi^{2}$ test for heterogeneity was not significant, with heterogeneity considered not important $\left(x^{2}=1.19, p=0.76\right.$, $\mathrm{I}^{2}=0 \%$ ). No additional studies utilising midwife-led models of care reported on planned CS as an outcome.

\section{Unplanned caesarean section}

Four studies ( $n=5937$ participants) were included in a meta-analysis comparing midwife-led models of care with a comparator group for unplanned CSs, with results indicating no significant differences between treatment conditions (average RR 0.87, 95\% CI 0.73 to 1.03) (Table 2). The $\chi^{2}$ test for heterogeneity was not significant, with heterogeneity considered not important to moderate $\left(\chi^{2}=4.55, p=0.21\right.$, $\left.\mathrm{I}^{2}=34 \%, \quad \mathrm{Tau}^{2}=0.01\right)$. No additional studies utilising midwife-led models of care reported on unplanned CS as an outcome.

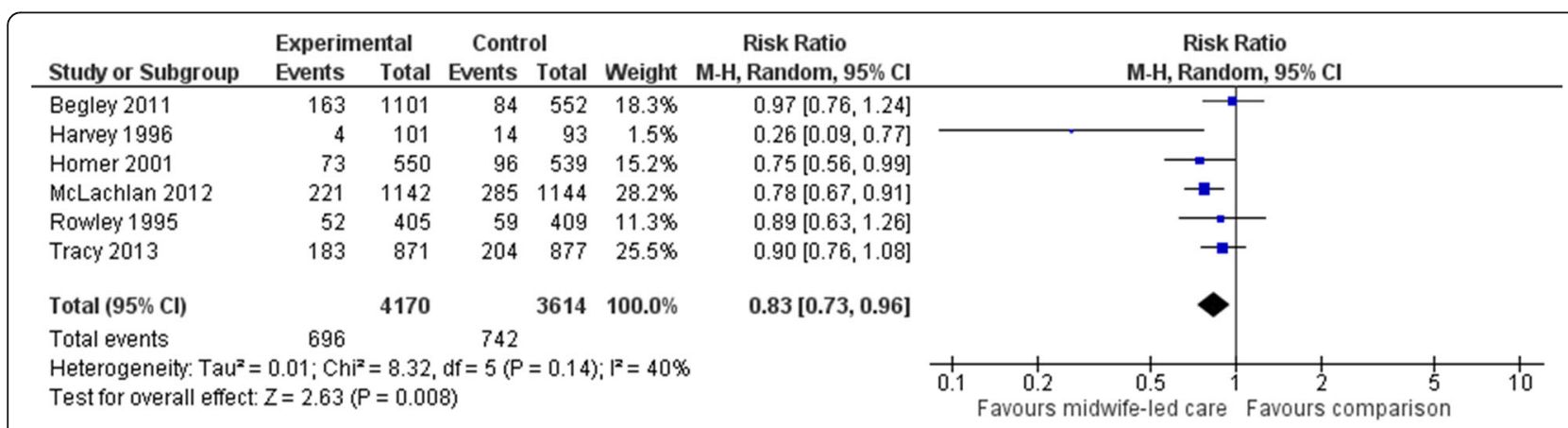

Fig. 2 Forest plot for the outcome 'overall caesarean section' in the selected RCTs, comparing midwife-led models of care (implemented across pregnancy, labour and birth, and the postnatal period) with standard care 


\begin{tabular}{|c|c|c|c|c|c|c|c|c|}
\hline Study or Subgroup & $\begin{array}{l}\text { Experim } \\
\text { Events }\end{array}$ & Experimental & \multicolumn{2}{|c|}{ Control } & Weight & $\begin{array}{c}\text { Risk Ratio } \\
\text { M-H, Random, } 95 \% \mathrm{Cl}\end{array}$ & \multicolumn{2}{|c|}{$\begin{array}{c}\text { Risk Ratio } \\
\text { M-H, Random, } 95 \% \mathrm{Cl}\end{array}$} \\
\hline Homer 2001 & 21 & 550 & 34 & 539 & $15.3 \%$ & $0.61[0.36,1.03]$ & T & F \\
\hline McLachlan 2012 & 35 & 1142 & 40 & 1144 & $21.7 \%$ & $0.88[0.56,1.37]$ & & \\
\hline Rowley 1995 & 21 & 405 & 26 & 409 & $13.8 \%$ & $0.82[0.47,1.43]$ & & \\
\hline Tracy 2013 & 69 & 871 & 94 & 877 & $49.2 \%$ & $0.74[0.55,0.99]$ & & \\
\hline Total $(95 \% \mathrm{Cl})$ & & 2968 & & 2969 & $100.0 \%$ & $0.75[0.61,0.93]$ & & \\
\hline Total events & 146 & & 194 & & & & & \\
\hline $\begin{array}{l}\text { Heterogeneity: Taúz } \\
\text { Test for overall effect }\end{array}$ & $\begin{array}{l}=0.00 ; \mathrm{Chi}^{2} \\
Z=2.66(\mathrm{~F}\end{array}$ & $\begin{array}{l}=1.19 \\
=0.008\end{array}$ & $\begin{array}{l}d f=3(P= \\
8)\end{array}$ & $=0.76)$ & $F^{2}=0 \%$ & & $\begin{array}{cc}1.5 & 0.7 \\
0.5 & 1 \\
\text { Favours midwife-led care } & 1\end{array}$ & $\begin{array}{cc}1.5 & 1 \\
1.5 & 2 \\
\text { Favours comparison }\end{array}$ \\
\hline
\end{tabular}

\section{Induction of labour}

Five studies ( $n=5498$ participants) were included in a meta-analysis comparing midwife-led models of care with a comparator group for induction of labour, with results indicating no significant differences between treatment conditions (average RR 0.91, 95\% CI 0.79 to 1.04) (Table 2). The $x^{2}$ test for heterogeneity was not significant, with heterogeneity considered not important to moderate $\left(\mathrm{X}^{2}=6.06, p=0.19, \mathrm{I}^{2}=34 \%\right.$, Tau $\left.{ }^{2}=0.01\right)$. No additional studies utilising midwife-led models of care reported on induction of labour as an outcome.

\section{Epidural use}

Six studies ( $n=7601$ participants) were included in a metaanalysis comparing midwife-led models of care with a comparator group for epidural use. Results indicated that the difference between midwife-led care and the comparator condition was not significant, albeit approaching significance (average RR $0.89,95 \%$ CI 0.79 to $1.00, p=0.05$ ) (Table 2). The $x^{2}$ test for heterogeneity was significant, with heterogeneity considered moderate to substantial $\left(x^{2}=\right.$ 10.82, $p=0.06, \mathrm{I}^{2}=54 \%$, Tau ${ }^{2}=0.01$ ). No additional studies utilising midwife-led models of care reported on epidural use as an outcome.

\section{Labour augmentation}

The outcome of labour augmentation incorporates the use of intravenous oxytocin and artificial rupture of the membranes (amniotomy) to increase the frequency, duration and intensity of contractions after the onset of spontaneous labour [41]. Five studies ( $n=5498$ participants) were included in a meta-analysis comparing midwife-led models of care with a comparator group for labour augmentation. Results indicated that the difference between midwife-led care and the comparator condition was not significant (average RR $0.97,95 \%$ CI 0.73 to 1.29 ) (Table 2). The $X^{2}$ test for heterogeneity was significant, with heterogeneity considered considerable $\left(x^{2}=48.99, p<0.00001, \mathrm{I}^{2}=92 \%\right.$, Tau $\left.{ }^{2}=0.09\right)$ . No additional studies utilising midwife-led models of care reported on labour augmentation as an outcome.

\section{Instrumental vaginal delivery}

The outcome of instrumental vaginal delivery encompasses the utilisation of either forceps or a vacuum device to assist in the vaginal delivery of a foetus [42]. Four studies ( $n=6776$ participants) were included in a metaanalysis comparing midwife-led models of care with a comparator group for instrumental vaginal delivery. Results indicated that the difference between midwife-led care and the comparator condition was not significant (average RR 0.96, 95\% CI 0.86 to 1.07) (Table 2). The $x^{2}$ test for heterogeneity was not significant, with heterogeneity considered not important $\left(\chi^{2}=1.82, p=0.61\right.$, $\left.\mathrm{I}^{2}=0 \%\right)$. No additional studies utilising midwife-led models of care reported on instrumental vaginal delivery as an outcome.

\section{Episiotomy}

Six studies ( $n=6816$ participants) were included in a metaanalysis comparing midwife-led models of care with a comparator group for episiotomy. Women allocated to midwife-led care were, on average, less likely to experience an episiotomy (average RR 0.84, 95\% CI 0.74 to 0.95 ) (Fig. 4) . The $\chi^{2}$ test for heterogeneity was not significant, with heterogeneity considered not important $(x 2=6.19, p=0.29$, $\mathrm{I}^{2}=19 \%$ ). Narratively, one RCT that compared labour and birth care provided by midwives in a birth centre with labour and birth care provided in a labour ward found that women who were allocated to midwife-led care received significantly fewer episiotomies (I: 24/222; C: $87 / 246$; $p<0.0005)$ [39].

\section{Admission during pregnancy}

Four studies ( $n=5304$ participants) were included in a meta-analysis comparing midwife-led models of care with a comparator group for admission during pregnancy. Results indicated that the difference between midwife-led care and the comparator condition was not significant (average RR 0.94, 95\% CI 0.80 to 1.11) (Table 2). The $\mathrm{X}^{2}$ test for heterogeneity was significant, with heterogeneity considered moderate to substantial $\left(\chi^{2}=7.06, p=0.07\right.$, 


\begin{tabular}{|c|c|c|c|c|c|c|c|c|}
\hline \multirow[b]{2}{*}{ Study or Subgroup } & \multicolumn{2}{|c|}{ Experimental } & \multicolumn{2}{|c|}{ Control } & \multirow[b]{2}{*}{ Weight } & \multirow{3}{*}{$\begin{aligned} \begin{array}{c}\text { Risk Ratio } \\
\text { M-H, Random, } 95 \% \mathrm{Cl}\end{array} \\
0.93[0.70,1.23]\end{aligned}$} & \multirow{2}{*}{\multicolumn{2}{|c|}{$\begin{array}{c}\text { Risk Ratio } \\
\text { M-H, Random, } 95 \% \mathrm{Cl}\end{array}$}} \\
\hline & Events & Total & Events & Total & & & & \\
\hline Begley 2011 & 126 & 1101 & 68 & 552 & $15.6 \%$ & & $\longrightarrow$ & - \\
\hline Hawey 1996 & 15 & 97 & 26 & 79 & $4.4 \%$ & $0.47[0.27,0.82]$ & - & \\
\hline Homer 2001 & 63 & 550 & 66 & 539 & $12.0 \%$ & $0.94[0.68,1.29]$ & $\longrightarrow$ & \\
\hline McLachlan 2012 & 208 & 901 & 238 & 811 & $34.3 \%$ & $0.79[0.67,0.92]$ & $\longrightarrow$ & \\
\hline Rowley 1995 & 46 & 405 & 56 & 409 & $9.7 \%$ & $0.83[0.58,1.19]$ & & \\
\hline Tracy 2013 & 135 & 693 & 146 & 679 & $24.1 \%$ & $0.91[0.74,1.12]$ & $\rightarrow-$ & - \\
\hline Total $(95 \% \mathrm{Cl})$ & & 3747 & & 3069 & $100.0 \%$ & $0.84[0.74,0.95]$ & & \\
\hline Total events & 593 & & 600 & & & & & \\
\hline \multicolumn{7}{|c|}{$\begin{array}{l}\text { Heterogeneity: } \operatorname{Tau}^{2}=0.00 ; \mathrm{Chi}^{2}=6.19, \mathrm{df}=5(P=0.29) ; \mathrm{l}^{2}=19 \% \\
\text { Test for overall effect: } Z=2.87(P=0.004)\end{array}$} & $\begin{array}{ccc}1 & 1 \\
0.5 & 0.7 & 1 \\
\text { Favours midwife-led care }\end{array}$ & $\begin{array}{c}1.5 \frac{1}{2} \\
\text { Favours comparison }\end{array}$ \\
\hline
\end{tabular}

$\left.\mathrm{I}^{2}=58 \%, \quad \mathrm{Tau}^{2}=0.02\right)$. No additional studies utilising midwife-led models of care reported on admission during pregnancy as an outcome.

\section{Apgar scores (< 7 at 5 min)}

Three studies ( $n=4711$ participants) were included in a meta-analysis comparing midwife-led models of care with a comparator group for Apgar scores of $<7$ at $5 \mathrm{~min}$. Results indicated that the difference between midwife-led care and the comparator condition was not significant (average RR 0.94, 95\% CI 0.66 to 1.33) (Table 2). The $\chi^{2}$ test for heterogeneity was not significant, with heterogeneity considered not important $\left(\mathrm{X}^{2}=0.88, p=0.64, \mathrm{I}^{2}=0 \%\right)$. Narratively, five additional RCTs utilising midwife-led care reported on Apgar scores as an outcome. These RCTs could not be included in the meta-analysis due to either variations in Apgar scoring criteria (e.g. Apgar cut off < 8), or the implementation of interventions in pregnancy or labour and birth only. However, all five additional RCTs similarly observed no significant differences in Apgar scores between intervention and comparator conditions at $5 \mathrm{~min}[31-33,39,40]$.

\section{Admission to special care unit/neonatal intensive care unit} The meta-analysis for admission to a special care unit (SCU)/neonatal intensive care unit (NICU) incorporated data from five studies ( $n=6599$ participants). Three studies used the combined outcome of SCN/NICU admission [29, 30, 32]; one study used the outcome of NICU admission [34]; and one study used the outcome of SCN admission [31]. Results of the meta-analysis indicated that the difference between midwife-led care and the comparator condition was not significant (average RR 0.80, 95\% CI 0.62 to 1.04) (Table 2). The $\chi^{2}$ test for heterogeneity was significant, with heterogeneity considered moderate to substantial $\left(\chi^{2}=8.54, p=0.07, \mathrm{I}^{2}=53 \%, \mathrm{Tau}^{2}=0.04\right)$.

\section{Narrative synthesis of secondary outcomes}

A number of additional secondary outcomes of interest could not be included in a meta-analysis of midwife-led models of care. Birthweight and neonatal mortality were assessed within three studies utilising midwife-led care throughout pregnancy, labour and birth, and postnatal periods. Reporting of outcomes varied, preventing conduct of a meta-analysis, however all three studies found no significant differences between midwife-led care and the comparator condition [29, 34]. Postpartum haemorrhage was assessed in two studies (means of measurement not specified), both of which found no significant differences [31, 33]. Two studies reported on postnatal stay (1 day or less; and $0-2$ days) as an outcome, with both studies finding that women experiencing midwife-led care were significantly more likely to have shorter postnatal stays [30, 31]. The outcomes of estimated blood loss and perineal trauma were reported within four studies each, however outcome categories varied. Only one of the four studies, an RCT comparing midwife-led labour and birth care in a birthing suite, with labour and birth care in a labour ward, observed fewer 3rd and 4th degree perineal tears among women allocated to midwife-led care [39]. Differences between treatment groups in the four studies reporting on estimated blood loss were predominantly not significant (one blood loss category, <500 ml, displayed significance in one study [30]). Lastly, in the two studies reporting on continuous electronic foetal monitoring (EFM) as an outcome, one study observed a significant reduction in risk of continuous EFM for women allocated to the midwife-led group [31].

\section{Continuous midwifery care \\ Overall caesarean section}

Three studies ( $n=7428$ participants) were included in a meta-analysis comparing continuous midwifery care during labour and birth with a comparator group for overall CS, including planned and unplanned CSs. Results indicated that the difference between continuous midwifery care and the comparator condition was not significant (average RR $0.85,95 \%$ CI 0.59 to 1.23 ) (Table 2). The $\chi^{2}$ test for heterogeneity was not significant, with heterogeneity considered moderate to substantial $\left(\chi^{2}=4.27, p=0.12, \mathrm{I}^{2}=53 \%\right.$, $\left.\mathrm{Tau}^{2}=0.06\right)$. 


\section{Narrative synthesis of secondary outcomes}

A number of additional secondary outcomes of interest could not be included in a meta-analysis of continuous midwifery care $(<3$ studies per outcome per intervention type). Two included studies $[35,36]$ similarly reported on instrumental vaginal delivery, epidural use, perineal tear, and neonatal admission to higher-level care/NICU, with both studies observing no significant differences between continuous midwifery care and comparator groups. Apgar scores of $<7$ at $5 \mathrm{~min}$ and birth weight were also reported in two included studies utilising continuous midwifery care $[36,37]$, with no significant differences found in either study. No differential treatment effects for use of oxytocin in labour were observed within two included studies [35, 37]. Only one RCT utilising continuous midwifery care reported on the outcomes of episiotomy, postpartum haemorrhage, continuous EFM, labour augmentation and neonatal mortality [36]. Significant differences (at the $p<$ 0.001 level) were only observed in one outcome; women in the continuous midwifery care group were less likely to have continuous EFM.

\section{Audit and feedback to promote implementation of evidence- based practice}

One included study utilising a cluster RCT design investigated the effects of a multifaceted 1.5-year intervention [27]. The intervention involved audits of CS indications, the provision of formal and informal feedback to health professionals, and implementation of best practices. Small, but significant reductions were observed in CS rate from pre-intervention to post-intervention periods among the intervention group compared with the control (adjusted OR for incremental change over time $0.90,95 \% \mathrm{CI} 0.80$ to $0.99)$. CS rate was significantly reduced among women with low-risk pregnancies (adj. Risk difference-1.7, 95\% CI -3.0 to -0.3 ), but not among high-risk pregnancies $(p=0.35)$. With regard to secondary outcomes, the intervention group also displayed reductions in major neonatal morbidity (adj. Risk difference, $-0.7,95 \%$ CI -1.3 to 0.1 ), and a smaller increase in minor neonatal morbidity compared to the control (adj. Risk difference - 1.7, 95\% CI -2.6 to -0.9 ). No significant differences were observed between groups with regard to changes in minor and major maternal morbidity [27].

\section{Labour assessment triage}

A RCT in Canada that examined the effects of labour assessment and triage at home versus telephone advice found no difference in CS rates between groups of healthy, nulliparous women (RR 1.12, 95\% CI 0.94 to 1.32) [38]. Differential treatment effects were not observed for the secondary outcomes of epidural use, labour augmentation, instrumental vaginal delivery, Apgar scores, and neonatal admission to higher-level care units.

\section{Hospital policy of mandatory second opinion for caesarean section}

One included study, a cluster RCT in Latin America, examined the effects of a mandatory second opinion policy for CS [26]. This study observed a small, but significant reduction in CS rates between matched hospitals (mean difference in rate change between matched hospitals: $-1.9,95 \%$ $\mathrm{CI}-3.8$ to -0.1 ). Secondary analysis by planned and unplanned CSs revealed a significant difference in unplanned CS rates between matched hospitals (RR -2.2, 95\% CI -4.3 to - 0.1), compared with no difference in planned CS rates (0.2, 95\% CI -1.4 to 1.8$)$. The implementation of the mandatory second opinion policy had no significant impact on the secondary outcomes of instrumental vaginal delivery, neonatal and maternal mortality, neonatal and perinatal mortality, neonatal and maternal admission to intensive care, and women's satisfaction with the care process [26].

\section{Hospital protocols for pregnancy complications}

One study, utilising a non-concurrent quasi-experimental controlled design, evaluated the effectiveness of protocols for pregnancy complications on CS rate [28]. The study was implemented in Iran and compared CS rates among women with pregnancy complications for periods before and after the initiation of new Ministry of Health and Medical Education protocols. The study found a significant increase in CS rate following the implementation of pregnancy complication protocols (CS rate: intervention phase: $67.8 \%$, control phase $48.8 \%, p=0.001$ ). With regard to secondary outcomes, duration of hospitalisation, number of specialist visits, and occurrence of complications post-discharge also significantly increased within the intervention phase compared with the control phase $(p=$ 0.001) [28].

\section{Risk of bias/quality appraisal}

Twelve RCTs clearly described the utilisation of genuine random sequence generation and were categorised as having a low risk of bias (Table 3). Two RCTs did not adequately detail their randomisation process and were classified as unclear $[37,39]$. Twelve RCTs were rated low risk of bias for allocation concealment, while the remaining two RCTs $[27,34]$ were judged as unclear due to insufficient information. Classifications for blinding of participants and personnel varied between studies; five RCTs were rated as high risk of bias [30, 31, 34-36]; four RCTs as unclear risk of bias [29, 32, 37, 40]; and the remaining five RCTs as low risk of bias [26, 27, 33, 38, 39]. The majority of included studies were judged as low risk of bias for blinding of outcome assessment $(\mathrm{k}=9)$. An additional four RCTs were rated as unclear [31, 32, 36, 37], while one RCT was classified as high risk of bias [34]. With regard to incomplete outcome data, most RCTs reported loss to follow-up rates of $<20 \%$ and utilised an intention-to-treat approach, and were 
Table 3 Risk of bias summary for studies utilising an RCT/cluster RCT design

\begin{tabular}{|c|c|c|c|c|c|c|c|}
\hline Study ID & $\begin{array}{l}\text { Random sequence } \\
\text { generation } \\
\text { (selection bias) }\end{array}$ & $\begin{array}{l}\text { Allocation } \\
\text { concealment } \\
\text { (selection bias) }\end{array}$ & $\begin{array}{l}\text { Blinding of participants } \\
\text { and personnel } \\
\text { (performance bias) }\end{array}$ & $\begin{array}{l}\text { Blinding of } \\
\text { outcome } \\
\text { assessment } \\
\text { (detection bias) }\end{array}$ & $\begin{array}{l}\text { Incomplete outcome } \\
\text { data (attrition bias) }\end{array}$ & $\begin{array}{l}\text { Selective reporting } \\
\text { (reporting bias) }\end{array}$ & $\begin{array}{l}\text { Other sources } \\
\text { of bias }\end{array}$ \\
\hline $\begin{array}{l}\text { Althabe } \\
{[2004]}\end{array}$ & + & + & + & + & + & + & $?$ \\
\hline $\begin{array}{l}\text { Begley } \\
{[2011]}\end{array}$ & + & + & - & $?$ & + & $?$ & + \\
\hline $\begin{array}{l}\text { Chaillet } \\
\text { [2015] }\end{array}$ & + & $?$ & + & + & + & + & $?$ \\
\hline $\begin{array}{l}\text { Chambliss } \\
\text { [1992] }\end{array}$ & $?$ & + & + & + & + & $?$ & + \\
\hline $\begin{array}{l}\text { Gagnon } \\
\text { [1997] }\end{array}$ & + & + & - & + & + & $?$ & + \\
\hline Gu [2013] & + & + & $?$ & + & + & $?$ & + \\
\hline $\begin{array}{l}\text { Harvey } \\
\text { [1996] }\end{array}$ & + & + & $?$ & $?$ & + & $?$ & $?$ \\
\hline $\begin{array}{l}\text { Hodnett } \\
\text { [2002] }\end{array}$ & + & + & - & $?$ & + & $?$ & + \\
\hline $\begin{array}{l}\text { Homer } \\
\text { [2001] }\end{array}$ & + & + & + & + & + & + & + \\
\hline $\begin{array}{l}\text { Janssen } \\
{[2006]}\end{array}$ & + & + & + & + & + & $?$ & $?$ \\
\hline $\begin{array}{l}\text { Kashanian } \\
{[2010]}\end{array}$ & $?$ & + & $?$ & $?$ & + & $?$ & + \\
\hline $\begin{array}{l}\text { McLaughlin } \\
\text { [2012] }\end{array}$ & + & + & $?$ & + & + & $?$ & + \\
\hline $\begin{array}{l}\text { Rowley } \\
\text { [1995] }\end{array}$ & + & $?$ & - & - & $?$ & $?$ & + \\
\hline Tracy [2013] & + & + & - & + & + & + & + \\
\hline
\end{tabular}

Note: Low risk of bias (+); High risk of bias (-); Unclear risk of bias (?)

subsequently judged as low risk of bias; one RCT did not provide sufficient information and was rated as unclear [34]. The majority of RCTs $(\mathrm{k}=10)$ were classified as unclear for selective outcome reporting because protocols were not available to determine if all outcome data collected were reported. Additionally, no potential other sources of bias were identified within most included studies $(\mathrm{k}=10)$.

The global rating for the one study utilising a quasiexperimental controlled design [28] was classified as 'weak', due to the occurrence of two or more components rated as 'weak'. Specifically, the selection bias, confounders, and blinding components were rated as 'weak'; the study design and data collection method components were rated as 'moderate'; and the withdrawals and dropouts component rated as 'strong'.

\section{Discussion}

This systematic review including meta-analyses synthesised published evidence and quantified the effects of a subset of maternity service organisational interventions on overall, planned, and unplanned CSs, in addition to a suite of relevant secondary birth outcomes. Results of the separate meta-analyses indicated that women allocated to midwifeled models of care implemented across pregnancy, labour and birth and postnatal periods were, on average, less likely to experience CS (overall), planned CS, and episiotomy compared with women allocated to usual care.

For the meta-analysis of midwife-led models of care, the significant reduction in risk of CS observed in the present review is inconsistent with that observed in a Cochrane review of midwife-led continuity models. No differences between groups were observed in the Cochrane review for overall CS [15]. This discrepancy in effects may largely be attributed to the differences in eligibility criteria between reviews. Unlike the Cochrane review, the present study tightly restricted eligibility to interventions with a primary aim of decreasing CSs. It is therefore not surprising that midwife-led models of care specifically designed to improve rates of physiological birth displayed significant risk reductions in CS rates, when compared to midwife-led models with varied aims. Consistent across both reviews however, was a significant finding for the secondary outcome of episiotomy, with comparable significant risk ratios observed in both 
reviews (RRs of 0.84). No comparison can be made between reviews for the outcome of planned CS because it was not included as an outcome of interest in the Cochrane review.

The non-significant meta-analysis finding for the effect of continuous midwifery support on overall CS in the present review also differs with that observed in a Cochrane review of continuous one-to-one labour support. The Cochrane review found that women allocated to continuous one-toone support were less likely to have a CS (low-quality evidence) [16]. Considerable variations in eligibility criteria between reviews may explain this inconsistency. In contrast with the present study, the Cochrane review did not limit inclusion of studies to interventions designed to decease CS rates, and additionally included interventions that utilised informal support persons and doulas within interventions. As a result, the number of studies included in the metaanalysis in the present study was considerably smaller than that of the Cochrane review ( $k=3$ vs $k=24$ ).

The narrative synthesis of results has additionally identified audit and feedback, and a hospital policy of mandatory second opinion for CS, as interventions that may have potential to reduce CS rates. Both interventions types were utilised within only one included study, with both interventions resulting in small but significant differences favouring the intervention [26, 27]. Audit and feedback is defined in the literature as 'a summary of the clinical performance of healthcare provider(s) over a specified period of time' [43]. A previous meta-analysis of evidence-based organisational interventions found audit and feedback as effective in reducing CS rates [14]. However, a number of studies included in the previous meta-analysis were excluded in the present review due to differing classifications in study design, and the requirement for CS to be a primary outcome. With regard to the one study which implemented a hospital policy of mandatory second opinion for CS, secondary analysis revealed the improvement of CS rates were concentrated among unplanned CSs. This finding was not unexpected given the intervention was implemented during labour and birth [26].

A surprising result highlighted within one included study was the significant increase in CS rate observed following the implementation of Iranian Ministry of Health and Medical Education pregnancy complication protocols. The authors of this study acknowledged that the introduction of new protocols may have increased visibility of CS indications among health professionals. However, the authors also acknowledged that the developed protocols were not based on national evidence, but rather extracted from text books, and were therefore unlikely to reflect contemporary recommendations. Additionally, the researchers used a quasi-experimental design with a non-concurrent control group which limits the strength of the findings.
Maternity service organisational interventions are an important consideration given increasing rates of CSs and potential for maternal and perinatal morbidity. As reflected by the publication date of included studies (73\% of studies published 2001+), this is an expanding field of research with considerable scope for advancement. To increase the strength of future reviews on the topic, primary studies should strive for consistency in the reporting of outcomes. Studies included in this review varied in the reporting of routinely documented outcomes (e.g. Apgar score cut-offs, maternal blood loss) which subsequently restricted the number of studies included within specific meta-analyses. Future primary studies in the field should also be conducted and reported according to recognised reporting standards, such as the CONSORT statement. Furthermore, as this review has demonstrated, midwife-led models of care are associated with reductions in CS rates. This metaanalysis included interventions utilising caseload midwifery and team midwifery models. More interventions utilising either caseload or team midwifery approaches would enable separate meta-analyses to be performed for each organisational approach, and enable publication bias to be adequately investigated. Standardisation of terminology related to models of care such as midwife-led, caseload and continuity of midwife carer will provide clarification of future analysis and interpretation of results. Moreover, interventions utilising audit and feedback, as well as a hospital policy of mandatory second opinion for CS were identified in the present review as potentially effective for improving CS rates. To enable the quantification of intervention effects, additional primary studies that utilise these approaches alone or in combination are required. It may also be beneficial to study maternity care in OECD countries with low CS rates to identify and study innovations and interventions related to the organisation of care.

The conduct and reporting of this review adhered to the PRISMA statement and utilised systematic and rigorous methods based on Cochrane Collaboration recommendations. The inclusion criteria tightly restricted eligibility to studies that utilised maternity service organisational interventions with a primary outcome of CS. Language or setting restrictions were not applied to the search, which increased the opportunity to include non-English studies. However, no studies published in languages other than English were included in the final set of studies. Had the search strategy intentionally utilised alternative language/ country specific databases, the pool of included studies may have been larger, thereby increasing the generalisability of results to additional countries and health settings. An additional consideration that should be noted is the inability to assess publication bias and small study effects due to insufficient numbers of included studies. Planned subgroup analyses, using the Robson classification system, were also not possible due to the included studies being comprised of 
maternal participant groups that were either not described in sufficient detail, or encompassed a diverse mix of maternal groups (not separated in analysis). This prevented the determination of intervention effects for any of the ten Robson classification maternal groups.

\section{Conclusions}

The findings of this systematic review indicate that women allocated to midwife-led models of care implemented across pregnancy, labour, birth, and the postnatal period were, on average, less likely to experience CS (overall), planned CS, and episiotomy compared with women allocated to routine care. Additionally, the findings suggest audit and feedback, and a hospital policy of mandatory second opinion for CS, are potential interventions that may reduce CS rates. Further research is required to investigate these latter interventions. Given the findings of this review, maternity service leaders should consider the adoption of midwife-led models of care across the maternity episode within their organisations. On the basis that the majority of studies utilising midwife-led models included samples of women classified as being at low-risk for complications (or able to be classified as low-risk), the adoption of midwife-led models of care may be particularly suitable for this maternal group.

\section{Additional file}

Additional file 1: Example search strategy for MEDLINE. (DOCX 16 kb)

\section{Abbreviations}

Cl: Confidence Interval; CS: Caesarean Section; EFM: Electronic Foetal Monitoring; NICU: Neonatal Intensive Care Unit; OECD: Organisation for Economic Cooperation and Development; PRISMA: Preferred Reporting Items for Systematic reviews and Meta-Analyses; RCTs: Randomised Controlled Trials; RR: Risk Ratio; SCU: Special Care Unit

\section{Acknowledgments}

The authors would like to acknowledge Kerry Rigby for her assistance in the systematic search, screening process and quality appraisal of included studies. Appreciation is also extended to Health Science Librarians at Deakin University Louisa Sher and Rachel West, for their assistance with the design of the systematic search strategy.

\section{Authors' contributions}

$\mathrm{AMH}, \mathrm{CN}$ and $\mathrm{BK}$ conceived of the original research project. $\mathrm{AMH}$ and $\mathrm{CN}$ developed the protocol and $D B$ and $B K$ advised on protocol design. $A M H, R L, J C$ and $A C$ managed the systematic search. AC, RL, JC and AMH selected studies for inclusion (with consultation sought from CN and DB), extracted the data, and assessed risk of bias of included studies. AC performed the statistical analysis, interpreted data, and wrote the first draft of the manuscript. All authors critically revised the manuscript and approved the final version. All authors, internal and external, had full access to the study data and take responsibility for the integrity of the data and the accuracy of the data analysis. All authors have approved the final version of the manuscript.

\section{Authors' information}

Dr. Anna Chapman, PhD.

Post-doctoral Research Fellow, Deakin University.

Research Fellow, International Institute for Primary Health Care

Research, China.
Professor Cate Nagle, PhD

Professor of Nursing and Midwifery, James Cook University and Townsville Hospital and Health Service.

Professor Debra Bick, PhD.

Professor of Clinical Trials in Maternal Health, University of Warwick.

Dr. Rebecca Lindberg, PhD.

Lecturer in Population Nutrition, Institute for Physical Activity and Nutrition, Deakin University.

Professor Bridie Kent, PhD.

Executive Dean, Faculty of Health and Human Sciences, Plymouth University. Director of the Centre for Innovations in Health and Social Care (Plymouth

University): A Joanna Briggs Centre of Excellence.

Justin Calache.

Research Assistant, Deakin University.

Professor Alison Hutchinson, PhD.

Chair in Nursing, Centre for Quality and Patient Safety Research - Monash Health Partnership.

Co-Director, Centre for Quality and Patient Safety Research.

Co-Director, Centre for Innovation and Education in Aged Care.

Funding

This research was not supported by any funding.

Availability of data and materials

All data generated or analysed during this study are included in this published article and its additional file.

Ethics approval and consent to participate

Not applicable

\section{Consent for publication}

Not applicable

\section{Competing interests}

The authors declare that they have no competing interests. $\mathrm{CN}$ is an Associate Editor of BMC Pregnancy and Childbirth; she was not involved in the review of this manuscript. DB is supported by the National Institute for Health Research (NIHR) Collaboration for Leadership in Applied Health Research and Care South London.

\section{Author details}

${ }^{1}$ School of Nursing and Midwifery, Centre for Quality and Patient Safety Research, Deakin University, Geelong, VIC, Australia. ${ }^{2}$ Monash Medical Centre, Monash Health, Level 2 I Block, 246 Clayton Rd, Clayton 3168, VIC, Australia. ${ }^{3}$ Centre for Nursing and Midwifery Research, James Cook University, Townsville, Queensland, Australia. ${ }^{4}$ Townsville Hospital and Health Service, Townsville, Queensland, Australia. ${ }^{5}$ Warwick Clinical Trials Unit, Warwick Medical School, University of Warwick, Coventry, UK. ${ }^{6}$ Institute for Physical Activity and Nutrition, School of Exercise and Nutrition Sciences, Deakin University, Geelong, Victoria, Australia. ${ }^{7}$ Faculty of Health and Human Sciences, University of Plymouth, Plymouth, Devon, UK.

Received: 29 August 2018 Accepted: 4 June 2019

Published online: 09 July 2019

\section{References}

1. World Health Organization. WHO statement on caesarean section rates. Geneva: World Health Organization; 2015.

2. Betran AP, Torloni MR, Zhang JJ, Gulmezoglu AM. WHO working group on caesarean section: WHO statement on caesarean section rates. BJOG : An international journal of obstetrics and gynaecology. 2016;123(5):667-70.

3. OECD: Key indicators. In: OECD health statistics 2018. 28 June 2018 edn; 2018.

4. Molina G, Weiser TG, Lipsitz SR, Esquivel MM, Uribe-Leitz T, Azad T, Shah N, Semrau K, Berry WR, Gawande AA, et al. Relationship between cesarean delivery rate and maternal and neonatal mortality. JAMA. 2015;314(21):2263-70.

5. Australian Commission on Safety and Quality in Health Care, Australian Institute of Health and Welfare: 3.4 caesarean section 20-34 years. In: The second Australian atlas of healthcare variation. Sydney: ACSQHC; 2017.

6. Bodner K, Wierrani F, Grunberger W, Bodner-Adler B. Influence of the mode of delivery on maternal and neonatal outcomes: a comparison between 
elective cesarean section and planned vaginal delivery in a low-risk obstetric population. Arch Gynecol Obstet. 2011;283(6):1193-8.

7. Prior E, Santhakumaran S, Gale C, Philipps LH, Modi N, Hyde MJ. Breastfeeding after cesarean delivery: a systematic review and meta-analysis of world literature. Am J Clin Nutr. 2012;95(5):1113-35.

8. Alkiaat A, Hutchinson M, Jacques A, Sharp MJ, Dickinson JE. Evaluation of the frequency and obstetric risk factors associated with term neonatal admissions to special care units. Aust N Z J Obstet Gynaecol. 2013;53(3):277-82.

9. Petrou S, Khan K. An overview of the health economic implications of elective caesarean section. Appl Health Econ Health Policy. 2013;11(6):561-76.

10. OECD. Health at a glance 2017: OECD indicators. Paris: OECD Publishing; 2017.

11. Khunpradit S, Tavender E, Lumbiganon P, Laopaiboon M, Wasiak J, Gruen RL. Non-clinical interventions for reducing unnecessary caesarean section. Cochrane Database Syst Rev. 2011;6:CD005528.

12. Althabe F, Belizán JM. Rising trend in non-indicated caesarean deliveries: can the trend be reversed? Paediatr Perinat Epidemiol. 2017;31(4):263-6.

13. Cochrane effective practice and organisation of care (EPOC) group: EPOC website; 2015. http://epoc.cochrane.org/scope-our-work. Accessed 16 Oct 2017.

14. Chaillet N, Dumont A. Evidence-based strategies for reducing cesarean section rates: a meta-analysis. Birth. 2007;34(1):53-64.

15. Sandall J, Soltani H, Gates S, Shennan A, Devane D. Midwife-led continuity models versus other models of care for childbearing women. Cochrane Database Syst Rev. 2016;(4):CD004667.

16. Bohren MA, Hofmeyr GJ, Sakala C, Fukuzawa RK, Cuthbert A. Continuous support for women during childbirth. Cochrane Database Syst Rev. 2017;(7): CD003766.

17. Moher D, Liberati A, Tetzlaff J, Altman DG, Group P. Preferred reporting items for systematic reviews and meta-analyses: the PRISMA statement. BMJ. 2009:339:b2535.

18. Hutchinson AM, Nagle C, Kent B, Bick D, Lindberg R. Organisational interventions designed to reduce caesarean section rates: a systematic review protocol. BMJ Open. 2018;8(7):e021120.

19. Higgins JP, Altman DG, Sterne JA. Chapter 8: assessing risk of bias in included studies. In: JPT H, Green S, editors. vol. version 5.1.0 (updated march 2011): the Cochrane collaboration Cochrane handbook for systematic reviews of interventions; 2011.

20. National Collaborating Centre for methods and tools: quality assessment tool for quantitative studies; 2008. https://www.nccmt.ca/knowledgerepositories/search/14. Accessed 14 July 2018.

21. Review manager (RevMan). In., 5.3 edn. Copenhagen: the Nordic Cochrane Centre, the Cochrane collaboration; 2014.

22. Tufanaru C, Munn Z, Stephenson M, Aromataris E. Fixed or random effects metaanalysis? Common methodological issues in systematic reviews of effectiveness. International Journal of Evidence-Based Healthcare. 2015;13(3):196-207.

23. Higgins J, Green S: Cochrane Handbook for Systematic Reviews of Interventions. In. Version 5.1.0 [updated March 2011]: The Cochrane Collaboration; 2011.

24. Dickersin K, Berlin JA. Meta-analysis: state-of-the-science. Epidemiol Rev. 1992;14(1):154-76

25. Robson M, Murphy M, Byrne F. Quality assurance: the 10-group classification system (Robson classification), induction of labor, and cesarean delivery. Int J Gynecol Obstet. 2015;131(S1):S23-7.

26. Althabe F, Belizan JM, Villar J, Alexander S, Bergel E, Ramos S, Romero M Donner A, Lindmark G, Langer A, et al. Mandatory second opinion to reduce rates of unnecessary caesarean sections in Latin America: a cluster randomised controlled trial. Lancet. 2004;363(9425):1934-40.

27. Chaillet N, Dumont A, Abrahamowicz M, Pasquier J-C, Audibert F, Monnier P, Abenhaim HA, Dubé E, Dugas $M$, Burne $R$, et al. A cluster-randomized trial to reduce cesarean delivery rates in Quebec. N Engl J Med. 2015;372(18):1710-21.

28. Yavangi M, Sohrabi MR. Alishahi Tabriz a: effect of Iranian Ministry of Health protocols on cesarean section rate: a quasi-experimental study. Journal of Research in Health Sciences. 2013;13(1):48-52.

29. McLachlan H, Forster D, Davey M, Farrell T, Gold L, Biro M, Albers L, Flood $M$, Oats J, Waldenström U. Effects of continuity of care by a primary midwife (caseload midwifery) on caesarean section rates in women of low obstetric risk: the COSMOS randomised controlled trial. BJOG Int J Obstet Gynaecol. 2012;119(12):1483-92.

30. Tracy SK, Hartz DL, Tracy MB, Allen J, Forti A, Hall B, White J, Lainchbury A Stapleton H, Beckmann M, et al. Caseload midwifery care versus standard maternity care for women of any risk: M@NGO, a randomised controlled trial. Lancet. 2013;382(9906):1723-32.
31. Begley C, Devane D, Clarke M, McCann C, Hughes P, Reilly M, Maguire R, Higgins S, Finan A, Gormally S, et al. Comparison of midwife-led and consultant-led care of healthy women at low risk of childbirth complications in the Republic of Ireland: a randomised trial. BMC Pregnancy and Childbirth. 2011;11(1):85

32. Harvey $S$, Jarrell J, Brant $R$, Stainton $C$, Rach D. A randomized, controlled trial of nurse-midwifery care. Birth. 1996;23(3):128-35.

33. Homer CSE, Davis GK, Brodie PM, Sheehan A, Barclay LM, Wills J, Chapman MG. Collaboration in maternity care: a randomised controlled trial comparing community-based continuity of care with standard hospital care BJOG Int J Obstet Gynaecol. 2001;108(1):16-22.

34. Rowley MJ, Hensley MJ, Brinsmead MW, Wlodarczyk JH. Continuity of care by a midwife team versus routine care during pregnancy and birth: a randomised trial. Med J Aust. 1995;163(6):289-93.

35. Gagnon AJ, Waghorn K, Covell C. A randomized trial of one-to-one nurse support of women in labor. Birth. 1997;24(2):71-7.

36. Hodnett ED, Lowe NK, Hannah ME, et al. Effectiveness of nurses as providers of birth labor support in north american hospitals: A randomized controlled trial. JAMA. 2002;288(11):1373-81.

37. Kashanian M, Javadi F, Haghighi MM. Effect of continuous support during labor on duration of labor and rate of cesarean delivery. Int J Gynecol Obstet. 2010;109(3):198-200.

38. Janssen PA, Still DK, Klein MC, Singer J, Carty EA, Liston RM, Zupancic JA. Early labor assessment and support at home versus telephone triage: a randomized controlled trial. Obstet Gynecol. 2006;108(6):1463-9.

39. Chambliss LR, Daly C, Medearis AL, Ames M, Kayne M, Paul R. The role of selection bias in comparing cesarean birth rates between physician and midwifery management. Obstet Gynecol. 1992;80(2):161-5.

40. Gu C, Wu X, Ding Y, Zhu X, Zhang Z. The effectiveness of a Chinese midwives' antenatal clinic service on childbirth outcomes for primipare: a randomised controlled trial. Int J Nurs Stud. 2013;50(12):1689-97.

41. World Health Organization. WHO recommendations for augmentation of labour. Geneva: WHO Press; 2014

42. Simms R, Hayman R. Instrumental vaginal delivery. Obstetrics, Gynaecology \& Reproductive Medicine. 2013;23(9):270-8.

43. Ivers $\mathrm{N}$, Jamtvedt G, Flottorp S, Young JM, Odgaard-Jensen J, French SD, O'Brien MA, Johansen M, Grimshaw J, Oxman AD. Audit and feedback: effects on professional practice and healthcare outcomes. Cochrane Database Syst Rev. 2012;(6):CD000259.

\section{Publisher's Note}

Springer Nature remains neutral with regard to jurisdictional claims in published maps and institutional affiliations.

Ready to submit your research? Choose BMC and benefit from

- fast, convenient online submission

- thorough peer review by experienced researchers in your field

- rapid publication on acceptance

- support for research data, including large and complex data types

- gold Open Access which fosters wider collaboration and increased citations

- maximum visibility for your research: over $100 \mathrm{M}$ website views per year

At $\mathrm{BMC}$, research is always in progress.

Learn more biomedcentral.com/submission 